Version of Record: https://www.sciencedirect.com/science/article/pii/S0003426618300192

Manuscript_2e9f862d54dff9069d45af193c24bf98

\title{
Bilateral adrenalectomy in Cushing's disease: altered long-term quality of life compared to other treatment options
}

Pauline Sarkis*, Muriel Rabilloud†, Jean-Christophe Lifante $\ddagger$, Anna Siamand§

, Emmanuel Jouanneau ๆ, Emmanuel Gay **, Philippe Chaffanjon††, ?Olivier Chabre , Gérald Raverot§§

*Hospices Civils de Lyon, Fédération d'endocrinologie, Groupement Hospitalier Est, 59, boulevard Pinel, 69677 Lyon,

+ Hospices Civils de Lyon, Service de Biostatistique et Bioinformatique, F-69003 Lyon, France; Université de Lyon, F-69000 Lyon, France; Université Lyon 1, F-69100 Villeurbanne, France; CNRS, UMR 5558,

Laboratoire de Biométrie et Biologie Evolutive, Equipe Biostatistique-Santé, F-69100 Villeurbanne, ¥Service de chirurgie générale digestive et endocrinienne, CH Lyon Sud, Hospices Civils de Lyon, 69495, Pierre Bénite,

Endocrinologie, Pavillon des Ecrins, Centre Hospitalier Universitaire de Grenoble, Cs 10217, 38043

Grenoble Cedex 9,

Il Hospices Civils de Lyon, Service de Neurochirurgie, Groupement Hospitalier Est, 59, boulevard Pinel, 69677 Lyon,

**Service de neurochirugie, Centre Hospitalier Universitaire, Grenoble, Grenoble Institut des Neurosciences, Centre de Recherche INSERM U836 UJF-CEA-CHU, Equipe 7, Bâtiment Edmond J Safra, Chemin Fortuné Ferrini, 38700 La Tronche,

++CHU Grenoble-Alpes, Département de Chirurgie Thoracique, Vasculaire et Endocrinienne, 38700 La

Tronche, Université Grenoble Alpes, LADAF-Laboratoire d'Anatomie Des Alpes Françaises, UFR de Médecine, 38700 La Tronche, and

§ Hospices Civils de Lyon, Fédération d'endocrinologie, Groupement Hospitalier Est, 59, boulevard Pinel, 69677 Lyon, Inserm U1052, CNRS UMR5286, Lyon I University, Signaling, metabolism and tumor progression, Cancer Center of Lyon, Centre Léon Bérard, Bat Cheney D- $5^{\text {th }}$ Floor, 28 rue Laennec 69008 Lyon, France. 


\section{Summary}

Objective Bilateral adrenalectomy (BADX) has become an important treatment of Cushing's disease (CD), especially when other treatment options have failed. The aim of this study was to evaluate the long-term quality of life (QoL) of patients having undergone BADX for $\mathrm{CD}$, in comparison to other therapeutic options.

Methods Thirty four patients with CD were identified in two French centers: 17 underwent BADX and the remaining 17 one or more of the following treatments: surgery, medical therapy or radiotherapy. Three questionnaires were filled in by each patient in order to evaluate their QoL: Short Form-36 Health Survey (SF-36), Cushing QoL questionnaire and Beck depression inventory (BDI).

Results The mean age of patients was $49.3 \pm 15.2$ years. Average time lapse between diagnosis and BADX was 6.1 years. Results from each questionnaire adjusted to age showed a lower QoL among patients who underwent BADX. These were significant in most aspects of the SF-36 questionnaire (bodily pain $\mathrm{p}<0.01$, general health $\mathrm{p}<0.01$, vitality $\mathrm{p}=<0.05$, social functioning $\mathrm{p}=<0.05)$, as well as in the Cushing QoL questionnaire $(\mathrm{p}<0.05)$ and BDI $(\mathrm{p}=<0.05)$. Adrenal insufficiency appeared to be the major predictor of poor QoL whatever their initial treatment.

Conclusions Despite their clinical remission, patients who undergo BADX appear to be at a greater risk of suffering an impaired QoL due to more prolonged period of time with imperfectly controlled hypercortisolism combined with definitive adrenal insufficiency. 


\section{Introduction}

Cushing's disease (CD) is the consequence of chronic exposure to glucocorticoids due to a pituitary adenoma. It comprises a large spectrum of signs and symptoms that cause an increased morbidity and mortality, and deterioration in quality of life (QoL)[1,2]. The first-line treatment option is surgical resection of the adenoma[3,4]. However, $31 \%$ of patients with $\mathrm{CD}$ will require a secondary treatment option, either due to a failure by a first pituitary surgery to control the symptoms or due to recurrence[5].

Second-line treatment options are radiotherapy, medical treatment, repeated transsphenoidal surgery (TSS) and bilateral adrenalectomy $(\mathrm{BADX})[4]$. BADX is an increasingly used and essential treatment option. A recent study reported $18 \%$ of $\mathrm{BADX}$ in a large cohort of $\mathrm{CD}[6]$. Low morbidity and mortality rates have recently been reported for BADX, with respective medians of $18 \%$ and $3 \%[6]$. However, this treatment implies lifelong glucocorticoid substitution and the risk of developing Nelson's syndrome.

Patients in whom $\mathrm{CD}$ has been cured nevertheless have an altered QoL compared to the general populatio[2,7]. The same holds true for patients cured by BADX, where the few existing studies report an improved QoL in $82-89 \%$ of cases, that remains however reduced when compared to healthy control[8-10]. To our knowledge, no studies have been published that compare long-term outcome of patients' QoL following different treatment options; most studies have focused on biochemical outcome, and the few concerning QoL have mostly compared patients to a healthy population.

The objective of this study was to evaluate the long-term QoL of patients who underwent BADX for CD, and to compare it to other therapeutic options.

\section{Subjects and methods}

\section{Participants}

Patients of two French centers were sent a letter of information about our study together with three questionnaires and a request for further medical information, which they were asked to complete should they agree to participate. Patients had to be in remission or controlled by medical treatment. One of the centers recruited only BADX patients, which were all not cured after at least a transphenoidal surgery. They were 
identified using the endocrinology unit register, and were treated between 1990 and 2015. The other center recruited CD patients with all kinds of treatment: BADX patients were identified using the surgery unit register, where the procedures were performed, and patients who had other type of treatment were identified using the register of the Federation of Endocrinology, tertiary referral center. Patients were treated between 2000 and 2015. CD patients who did not have BADX could have been treated with only one transphenoidal surgery, several surgeries, radiotherapy or medical treatment. We excluded patients with missing data about initial information on the $\mathrm{CD}$, or absence of an accurate postal address. In total, 78 patients received the questionnaires. 36 patients gave written consent, 18 with BADX and 18 without. Of these, two were excluded: one for incomplete response and the other for late response after the analysis had started. Patients were assigned to one of two groups according to whether they underwent bilateral adrenalectomy or not. The other treatment options making up the second group of 17 patients were TSS, repeated or not, medical treatment, and radiotherapy. Some patients had undergone several treatments. Medical records were retrospectively reviewed and entered into a database. Patients also provided complementary information concerning their comorbidities, substitutive treatment, and eventual presentation of adrenal crisis, which was defined as the need for i.v. glucocorticoid administration. Nelson's syndrome was reported in patients treated for it by radiotherapy or surgery as testified in their medical records.

Duration of exposure was defined as the time lapse between diagnosis and remission. Remission from CD was defined by glucocorticoid substitution or 24-h urinary free cortisol excretion within normal limits, with several controls. Patients who were under medical treatment for their CD were considered stable if their 24-h urinary free cortisol excretion was within normal limits. To evaluate QoL in regards to duration of exposure and of remission, we divided each of the two treatment groups according to duration of exposure and of remission: the three subgroups for exposure duration were: $<1$ year, 1-4 years and $\geq 5$ years, based on the assumption that patients affected less than a year by CD would have a better QoL. For remission duration, the subgroups were: < 2 years, 2-4 years, $\geq 5$ years. The lower limit of 2 years was based on reported long time lapse before symptom resolution after surgery[11]. The upper limit was chosen for both groups based on the assumption that no difference in QoL will be measurable between 5 years of remission or exposure and longer durations. 


\section{Measures}

The filled in questionnaires that were sent back from those patients who agreed to participate, using a prepaid envelope provided with the letter sent to their home, were the following:

- A generic health-related QoL questionnaire: the Short Form-36 Health Survey (SF-36)[12]. This consists of 36 items and 8 subscales: 4 constituting a physical score (physical functioning, role physical i.e. role limitations due to physical health, bodily pain and general health perception) and 4 a mental score (vitality, social functioning, role emotional i.e. role limitations due to emotional problems, and mental health). The score ranges from 0 to 100,100 being the best possible QoL.

- A disease-specific questionnaire: the Cushing QoL questionnaire (CushQoL)[13]. This consists of 12 items giving a total score for conversion onto a 0-100 scale; the higher scores being defined as the best QoL. To fill in this questionnaire, patients had to consider the past 4 weeks and how CD had interfered with their usual activities. It covers several concepts including healing and pain, mood and selfconfidence, daily activities, social concerns and physical concerns, memory and worry about health in the future.

- A depression-specific questionnaire: Beck depression inventory (BDI)[14]. This consists of 21 items giving a total score, with 17 representing the lower limit for depression symptoms.

\section{Statistical Analysis}

The characteristics of the patients were described by the mean and standard deviation for quantitative characteristics and by the absolute and relative frequencies in each category for qualitative characteristics. The Qol scores were described by the quartiles. The comparisons were carried out using the non-parametric test of Mann-Whitney for the quantitative variables and using the chi-squared or the Fisher exact test for the qualitative variables. Furthermore, using linear regression method, influence of age was investigated.

All the statistical analysis was carried out using the statistical software SPSS for Windows, version 19.0. Statistical significance was defined by a p-value $<0.05$. 


\section{Results}

\section{Patients' characteristics}

We identified 34 patients with CD. Figure 1 summarizes the treatments patients received. Seventeen patients underwent BADX, which was never as a first intention treatment. The average time between diagnosis and BADX was 6.1 years. The 17 other patients were treated by one or several of the following treatments: TSS, repeated or not, medical treatment, radiotherapy.

The characteristics of patients with CD are shown in Table 1. Twenty-six (76.5\%) of the 34 patients with CD were women. The mean age was $49.3 \pm 15.2$ years. While $24(70.6 \%)$ patients had received glucocorticoid substitution, acute adrenal insufficiency only occurred among patients having undergone BADX. Patients who underwent BADX had a longer duration of exposure $(6.1 \pm 7.2$ vs $1.3 \pm 2.3$ years, $p<0.01)$ and a longer duration of remission $(8.4 \pm 5,6$ vs $2.7 \pm 2.5$ years, $\mathrm{p}<0.01)$. Comorbidities (diabetes mellitus, hypertension, depressive syndrome, osteoporosis) were relatively similar between the two groups.

\section{Comparison of QoL between BADX and other treatment groups}

Table 2 shows the results of the different questionnaires addressed to the patients. Patients who underwent BADX had lower QoL scores in each questionnaire. This was significant in most aspects of the SF-36 questionnaire (bodily pain $\mathrm{p}<0.01$, general health $\mathrm{p}<0.01$, vitality $\mathrm{p}=<0.05$, social functioning $\mathrm{p}=<0.05$ ), as well as in the Cushing QoL questionnaire $(\mathrm{p}<0.05)$ and in the BDI $(\mathrm{p}=<0.05)$. Linear regression analysis did not find an influence of age on QoL.

\section{Comparison of QoL between different patient subgroups}

\section{Adrenal Insufficiency}

An interesting aspect was the presence of adrenal insufficiency. Every questionnaire revealed a lower QoL perceived by $\mathrm{CD}$ patients in remission but with adrenal insufficiency whatever their initial treatment, compared to patients in remission without adrenal insufficiency. The results were significant for SF-36 physical functioning $(\mathrm{p}=<0.05)$, role physical $(\mathrm{p}<0.01)$, general health $(\mathrm{p}=<0.05)$, social functioning $(\mathrm{p}=0.04)$, role emotional $(\mathrm{p}=<0.05)$ and BDI $(\mathrm{p}=<0.05)$. In linear regression analysis, only the item social functioning was no longer significant when adjusted to age. 
Moreover, no significant differences could be observed for each aspect of the questionnaire between patients who underwent BADX and those who did not but who had adrenal insufficiency except for SF-36 bodily pain (45 [35; 77.5] for BADX patients vs 100 [62.5; 100]), $\mathrm{p}=<0.05)$. When considering only those patients who did not undergo BADX and comparing those with or without adrenal insufficiency, no significant differences could be found between the groups, although the group without adrenal insufficiency had an overall better QoL, especially in the SF-36 items role physical $(100[81.2 ; 100]$ vs $25[0 ; 100])$ and role emotional $(100[100 ; 100]$ vs $66.7[0 ; 100])$.

Nelson's syndrome, adrenal crisis

Nelson's syndrome had a negative impact on QoL, revealed especially by the BDI questionnaire for which there was a significant difference between groups $(\mathrm{p}<0.05)$. In contrast, comparison of BADX patients who suffered of at least one adrenal crisis and those who did not showed no difference in QoL.

Exposure and remission duration, age, sex, radiotherapy, comorbidities.

We found no statistical significant differences in QoL in respect to duration of exposure, although a trend showing that shorter exposure time favored the QoL could be observed., especially in CushQoL (59 [54.5; 65.5] for the least exposed group vs 46 [31; 53] for the group exposed more than five years), bodily pain (80 [63.1; $100]$ vs $62.5[45 ; 87.5])$, general health $(52.1[44.8 ; 71.8]$ vs 41.7 [22.2; 62.5]) and social functioning (62.5 [35; 61.2] vs 50 [22.5; 50]). No significant differences in QoL were found with regards to remission duration. There were no significant differences in QoL between men and women, or with regards to radiotherapy treatment or the presence of comorbidities.

\section{Discussion}

While the QoL among CD patients in remission is known to be reduced compared to the general population, only a few studies have evaluated the role of treatment received in determining this QoL. In the present study, we compared 17 patients with $\mathrm{CD}$ who underwent BADX against those who underwent other treatment options to determine any differences in detrimental impact on QoL. In our study, patients who underwent BADX for CD 
had a lower QoL than patients who received another type of treatment. The difference in QoL between patients who had adrenal insufficiency compared to those who had not was also very strong, reaching significance in almost every aspect of each questionnaire. Unfortunately, the absence dedicated questionnaire evaluating the QoL of adrenal insufficiency (Addi-QoL) represent a major limitation of our study. Moreover, we were unable to show any significant differences with respect to remission and exposure duration. However, patients who had been less exposed to hypercorticism did tend to have a better QoL.

The second choice of treatment after TSS has failed is a challenging question. Among the several options, BADX is a very effective one, with an immediate effect on hypercorticism and, in the light of developments in laparoscopy, with very low morbidity and mortality. Our results show a greater negative impact of BADX on QoL compared to other treatment options. A few studies have also focused on QoL following BADX[8-10,15], using mostly the SF-36 questionnaire to assess and compare it to that of the general population. In comparative studies, we found similar or slightly lower SF-36 scores for BADX patients[8,15,16], adding weight to the representativeness of our population. In their study, Van Aken et al[16] compared against the general population the QoL of 58 patients in remission for CD, mostly treated by TSS with a mean follow-up of 13.4 years. Their patients also answered questionnaires, including SF-36, with a high response rate of 95\%. Apart from their CD population having a slightly better QoL than did ours, the strongest difference being for role physical which was much lower in our population $(54.4 \pm 43.3$ vs $65 \pm 41)$, our results were quite similar. The lower QoL found in our population can be explained by the higher number of patients in our study who received several treatments compared to theirs where most patients only had one TSS. Concordantly, we also did not find a relationship between duration of remission and QoL.

Our results indicate that patients having undergone BADX have a lower QoL compared to those having received other treatment options. Such a result could disfavor use of BADX as a second line treatment however, considering the lack of studies comparing QoL among patients with CD receiving different treatment, our result remains to be confirmed. Moreover it is important to note that only patient with severe Cushing disease, who failed to be controlled by TSS or any medical treatment and thus who had been exposed for a more prolonged period of time underwent BADX. In absence of BADX, those patients would have suffer from Cushing disease morbidity and mortality. Although not statistically different to impact QoL, a mean exposure duration was longer in BADX group compare to the other treatment group (6.1 \pm 7.2 vs $1.3 \pm 2.3$ years). We cannot exclude that it could affect the result. Should this duration be shortened, QoL may prove to be identical between the BADXtreated and other treatment groups of patients. This statement could not be proved in this study, probably due to 
the size of our population, but exposure duration is most likely a very strong predictive factor of QoL. Indeed, the difference between our two groups is high, (4.8 years) in disfavor of BADX patients. This longer exposition and its physical and psychological consequences, as well as the repeated treatment failures must play an important part in patient's QoL. We were also not able to show the impact of remission duration but the two groups were statistically different $(8.4 \pm 5,6$ vs $2.7 \pm 2.5$ years, $\mathrm{p}<0.01)$. Only Wagenmakers et al[7], who evaluated QoL in patients in long-term remission of Cushing's syndrome compared to an age and sex-matched control group, were able to identify a significant influence of remission duration. The discrepancy in our results may come from a larger population (123 patients) in the Wagenmakers et al study which was also divided up in a different manner (remission for 2-5 years, 5-10 years and 10 years or longer) to our study population (remission under 2 years, 2-5 years and 5 years or longer). They also raise the hypothesis that the cause of impaired QoL could be the previous period of long-standing exposure to high cortisol levels.

Most studies about QoL in patients with CD concern treatment with TSS. Long-term data on other types of treatment are lacking. Nelson et al[19] used the CushQoL questionnaire to report on patients with CD who participated in a phase 3 clinical trial designed to assess the safety and efficacy of pasireotide. The results at respectively months 6 and 12 showed a score of $41.1 \pm 20.4$ and $52.5 \pm 19$. In our study, patients who had

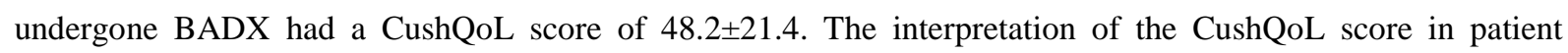
presenting adrenal with adrenal insufficiency may not be adequate. Ideally, such evaluation would be performed prospectively pre and prost operatively to evaluate the direct impact of the surgery on the CushQoL. However, secondary adrenal insufficiency will always introduce bias with specific symptoms associated with adrenal insufficiency in absence of good control group

One of the strengths of our study is the use of various questionnaires, including one general and two more specific ones: one concentrating on mental aspects and the other on disease-specific symptoms. While a few studies have evaluated long-term QoL of patients who underwent BADX, to our knowledge none directly compared these patients to those treated differently.

Our study also has some limitations. By its retrospective nature, it was impossible to achieve a standardized follow-up and some data were sometimes difficult to recover. Moreover the non BADX group included only $41 \%$ patients with adrenal insufficiency, which limitates the comparison. Other data like adrenal crisis were in some cases only based on patients' declarations which, while we did provide patients with a precise definition of the term "adrenal crisis" to limit any false statements, could represent a limitation. Another point is the fact that we only obtained the QoL perceived by the patients who agreed to participate in the study, which limits its 
significance. One could argue that patients who answered are the ones with a more altered QoL, leading to a selection bias. Moreover, as we mentioned before, BADX patients had an uncontrolled disease for a longer period, BADX treatment being the last line treatment, which might also interfere we the results. A prospective study should provide more precision, although considering the rarity of the disease and of the treatment, implementing such a prospective study will be challenging.

\section{Conclusion}

BADX is an essential treatment option for $\mathrm{CD}$, with high rates of success. However, BADX did not restore the QoL that we can expect for CD patient in remission. Glucocorticoid substitution seems to have an important role in this impairment, and may have a stronger impact than the type of treatment. Larger prospective studies are needed to evaluate the impact on QoL of BADX for CD.

Acknowledgements The authors wish to thank the French association "Surrénale" for the research grant and their contribution towards the development of this project. Thanks also to H. Lasolle for her help with the statistical analysis and Angloscribe for help with English proofing.

Declaration of interest The authors declare that they have no conflict of interest. 


\section{References}

[1] Feelders RA, Pulgar SJ, Kempel A, Pereira AM. The burden of Cushing's disease: clinical and health-related quality of life aspects. Eur J Endocrinol Eur Fed Endocr Soc 2012;167:311-26. doi:10.1530/EJE-11-1095.

[2] Alcalar N, Ozkan S, Kadioglu P, Celik O, Cagatay P, Kucukyuruk B, et al. Evaluation of depression, quality of life and body image in patients with Cushing's disease. Pituitary 2013;16:333-40. doi:10.1007/s11102012-0425-5.

[3] Lacroix A, Feelders RA, Stratakis CA, Nieman LK. Cushing's syndrome. Lancet Lond Engl 2015;386:913-27. doi:10.1016/S0140-6736(14)61375-1.

[4] Nieman LK, Biller BMK, Findling JW, Murad MH, Newell-Price J, Savage MO, et al. Treatment of Cushing's Syndrome: An Endocrine Society Clinical Practice Guideline. J Clin Endocrinol Metab 2015;100:2807-31. doi:10.1210/jc.2015-1818.

[5] Petersenn S, Beckers A, Ferone D, van der Lely A, Bollerslev J, Boscaro M, et al. Therapy of endocrine disease: outcomes in patients with Cushing's disease undergoing transsphenoidal surgery: systematic review assessing criteria used to define remission and recurrence. Eur J Endocrinol Eur Fed Endocr Soc 2015;172:R227-39. doi:10.1530/EJE-14-0883.

[6] Ritzel K, Beuschlein F, Mickisch A, Osswald A, Schneider HJ, Schopohl J, et al. Clinical review: Outcome of bilateral adrenalectomy in Cushing's syndrome: a systematic review. J Clin Endocrinol Metab 2013;98:3939-48. doi:10.1210/jc.2013-1470.

[7] Wagenmakers M a. EM, Netea-Maier RT, Prins JB, Dekkers T, den Heijer M, Hermus ARMM. Impaired quality of life in patients in long-term remission of Cushing's syndrome of both adrenal and pituitary origin: a remaining effect of long-standing hypercortisolism? Eur J Endocrinol Eur Fed Endocr Soc 2012;167:687-95. doi:10.1530/EJE-12-0308.

[8] Hawn MT, Cook D, Deveney C, Sheppard BC. Quality of life after laparoscopic bilateral adrenalectomy for Cushing's disease. Surgery 2002;132:1064-8; discussion 1068-9. doi:10.1067/msy.2002.128482.

[9] Thompson SK, Hayman AV, Ludlam WH, Deveney CW, Loriaux DL, Sheppard BC. Improved quality of life after bilateral laparoscopic adrenalectomy for Cushing's disease: a 10-year experience. Ann Surg 2007;245:790-4. doi:10.1097/01.sla.0000251578.03883.2f.

[10] Oßwald A, Plomer E, Dimopoulou C, Milian M, Blaser R, Ritzel K, et al. Favorable long-term outcomes of bilateral adrenalectomy in Cushing's disease. Eur J Endocrinol Eur Fed Endocr Soc 2014;171:209-15. doi:10.1530/EJE-14-0214.

[11] Sippel RS, Elaraj DM, Kebebew E, Lindsay S, Tyrrell JB, Duh Q-Y. Waiting for change: symptom resolution after adrenalectomy for Cushing's syndrome. Surgery 2008;144:1054-60; discussion 1060-1. doi:10.1016/j.surg.2008.08.024.

[12] Ware JE, Sherbourne CD. The MOS 36-item short-form health survey (SF-36). I. Conceptual framework and item selection. Med Care 1992;30:473-83.

[13] Webb SM, Badia X, Barahona MJ, Colao A, Strasburger CJ, Tabarin A, et al. Evaluation of health-related quality of life in patients with Cushing's syndrome with a new questionnaire. Eur J Endocrinol Eur Fed Endocr Soc 2008;158:623-30. doi:10.1530/EJE-07-0762.

[14] Beck AT, Ward CH, Mendelson M, Mock J, Erbaugh J. An inventory for measuring depression. Arch Gen Psychiatry 1961;4:561-71.

[15] Smith PW, Turza KC, Carter CO, Vance ML, Laws ER, Hanks JB. Bilateral adrenalectomy for refractory Cushing disease: a safe and definitive therapy. J Am Coll Surg 2009;208:1059-64. doi:10.1016/j.jamcollsurg.2009.02.054.

[16] van Aken MO, Pereira AM, Biermasz NR, van Thiel SW, Hoftijzer HC, Smit JWA, et al. Quality of life in patients after long-term biochemical cure of Cushing's disease. J Clin Endocrinol Metab 2005;90:327986. doi:10.1210/jc.2004-1375.

[17] Simon N, Castinetti F, Ouliac F, Lesavre N, Brue T, Oliver C. Pharmacokinetic evidence for suboptimal treatment of adrenal insufficiency with currently available hydrocortisone tablets. Clin Pharmacokinet 2010;49:455-63. doi:10.2165/11531290-000000000-00000.

[18] Barahona M-J, Sucunza N, Resmini E, Fernández-Real J-M, Ricart W, Moreno-Navarrete J-M, et al. Deleterious effects of glucocorticoid replacement on bone in women after long-term remission of Cushing's syndrome. J Bone Miner Res Off J Am Soc Bone Miner Res 2009;24:1841-6. doi:10.1359/jbmr.090505.

[19] Nelson LM, Forsythe A, McLeod L, Pulgar S, Maldonado M, Coles T, et al. Psychometric evaluation of the Cushing's Quality-of-Life questionnaire. The Patient 2013;6:113-24. doi:10.1007/s40271-013-0012-5. 
[20] Arlt W, Allolio B. Adrenal insufficiency. Lancet Lond Engl 2003;361:1881-93. doi:10.1016/S01406736(03)13492-7. 


\section{Figures and legends}

Fig. 1 Overview of the treatments received. MT: medical treatment; TSS: transsphenoidal surgery; RT: radiotherapy; BADX: bilateral adrenalectomy

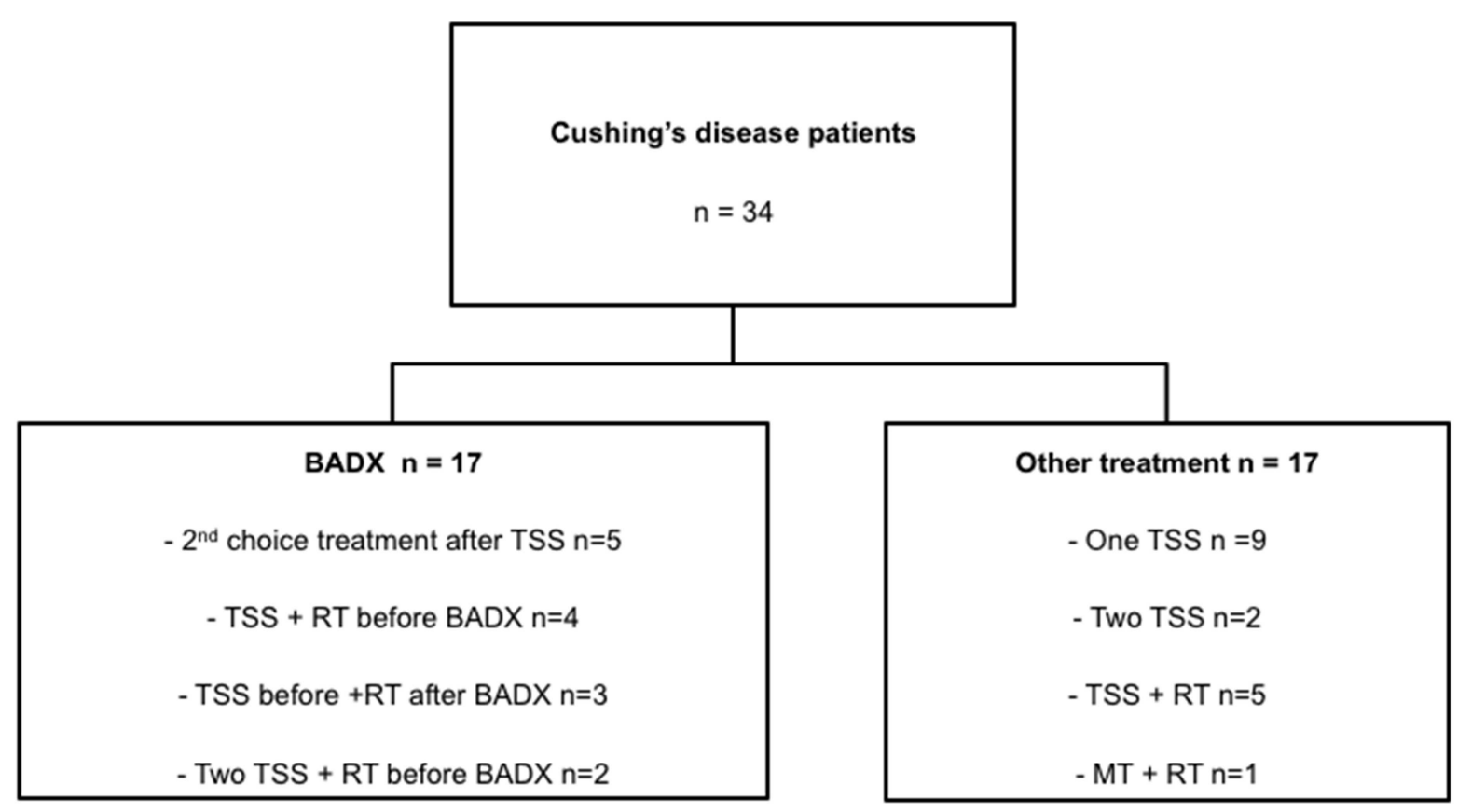

Table 1. Cushing's disease patients' characteristics

\begin{tabular}{lccc}
\hline & Bilateral ADX $(\mathrm{n}=17)$ & Other treatments $(\mathrm{n}=17)$ & $\mathrm{p}$-value \\
\hline Age (years) & $50 \pm 14.8$ & $48.5 \pm 16$ & 0.88 \\
Gender (female) & $14(82.3 \%)$ & $12(70.6 \%)$ & 0.69 \\
Duration of exposure (years) & $6.1 \pm 7.2$ & $1.3 \pm 2.3$ & $\mathbf{0 . 0 0 2}$ \\
Duration of remission (years) & $8.4 \pm 5,6$ & $2.7 \pm 2.5$ & $\mathbf{0 . 0 0 2}$ \\
Nelson's Syndrome & $5(29.4 \%)$ & 0 & $\mathbf{0 . 0 4}$ \\
Glucocorticoid substitution & $17(100 \%)$ & $7(41.2 \%)$ & $<\mathbf{0 . 0 0 1}$ \\
Acute adrenal insufficiency & $6(35 \%)$ & 0 & $\mathbf{0 . 0 2}$ \\
Comorbidities & $9(52.9 \%)$ & $10(58.8 \%)$ & 1 \\
Diabetes mellitus & 2 & 2 & \\
Hypertension & 2 & 5 & \\
Depressive syndrome & 4 & 2 & \\
Osteoporosis & 2 & 1 & \\
\hline
\end{tabular}

Bold values indicate statistical significance, $\mathrm{p}<0.05$

$\mathrm{ADX}=$ Adrenalectomy 
Table 2. Quality of life scores in Cushing's disease patients: bilateral ADX versus other treatments

\begin{tabular}{|c|c|c|c|}
\hline & $\begin{array}{c}\text { Bilateral ADX } \\
\quad(n=17) \\
\text { Median [Q1; Q3] }\end{array}$ & $\begin{array}{l}\text { Other treatments } \\
\qquad(\mathrm{n}=17) \\
\text { Median [Q1; Q3] }\end{array}$ & p-value \\
\hline \multicolumn{4}{|l|}{ SF-36 } \\
\hline Physical Functioning & $65[52.5 ; 77.5]$ & $90[65 ; 95]$ & 0.05 \\
\hline Role Physical & $25[0 ; 87.5]$ & $100[25 ; 100]$ & 0.05 \\
\hline Bodily Pain & $45[29 ; 79]$ & $90[67.5 ; 100]$ & 0.002 \\
\hline General Health & $42[27 ; 48]$ & $54[46 ; 73]$ & 0.005 \\
\hline Vitality & $35[20 ; 50]$ & $55[42.5 ; 65]$ & 0.02 \\
\hline Social Functioning & $50[50 ; 75]$ & 75 |62.5; 94] & 0.03 \\
\hline Role Emotional & $67[33 ; 100]$ & $100[67 ; 100]$ & 0.13 \\
\hline Mental Health & $56[48 ; 72]$ & $60[52 ; 74]$ & 0.62 \\
\hline Cush QoL & $44[31 ; 68.5]$ & $65[54 ; 74]$ & 0.02 \\
\hline BDI & $16[9.5: 24]$ & $9[4 ; 14]$ & 0.02 \\
\hline
\end{tabular}

Bold values indicate statistical significance, $\mathrm{p}<0.05$

SF-36 Short Form-36 Health Survey, Cush QoL Cushing quality of life questionnaire, BDI Beck depression inventory 